\title{
Early brain biomarkers of post-traumatic seizures: initial report of the multicentre epilepsy bioinformatics study for antiepileptogenic therapy (EpiBioS4Rx) prospective study
}

\author{
Evan S. Lutkenhoff, ${ }^{1,2}$ Vikesh Shrestha, ${ }_{1}^{2}$ Jesus Ruiz Tejeda, ${ }^{2}$ Courtney Real, ${ }^{2}$ \\ David L. McArthur, ${ }^{2}$ Dominique Duncan, ${ }^{3}$ Marianna La Rocca, ${ }^{3}$ Rachael Garner, ${ }^{3}$ \\ Arthur W. Toga, ${ }^{3}$ Paul M. Vespa, ${ }^{2,4}$ Martin M. Monti $10,1,2,5$ for the EpiBioS4Rx Study \\ Group
}

\begin{abstract}
- Additional material is published online only. To view please visit the journal online (http://dx.doi.org/10.1136/ jnnp-2020-322780).
\end{abstract}

For numbered affiliations see end of article.

\section{Correspondence to} Martin M. Monti, Psychology, University of California Los Angeles, Los Angeles, CA 90095, USA; monti@psych. ucla.edu

Received 10 January 2020 Revised 22 June 2020 Accepted 8 July 2020 Published Online First 26 August 2020

\section{Check for updates}

(C) Author(s) (or their employer(s)) 2020. No commercial re-use. See rights and permissions. Published by BMJ.

To cite: Lutkenhoff ES, Shrestha V, Ruiz Tejeda J, et al. J Neurol Neurosurg Psychiatry

2020:91:1154-1157.

\begin{abstract}
Background Traumatic brain injury (TBI) causes early seizures and is the leading cause of post-traumatic epilepsy. We prospectively assessed structural imaging biomarkers differentiating patients who develop seizures secondary to TBI from patients who do not.

Design Multicentre prospective cohort study starting in 2018. Imaging data are acquired around day 14 postinjury, detection of seizure events occurred early (within 1 week) and late (up to 90 days post-TBI).

Results From a sample of 96 patients surviving moderate-to-severe TBI, we performed shape analysis of local volume deficits in subcortical areas (analysable sample: 57 patients; 35 no seizure, 14 early, 8 late) and cortical ribbon thinning (analysable sample: 46 patients; 29 no seizure, 10 early, 7 late). Right hippocampal volume deficit and inferior temporal cortex thinning demonstrated a significant effect across groups. Additionally, the degree of left frontal and temporal pole thinning, and clinical score at the time of the MRI, could differentiate patients experiencing early seizures from patients not experiencing them with $89 \%$ accuracy.

Conclusions and relevance Although this is an initial report, these data show that specific areas of localised volume deficit, as visible on routine imaging data, are associated with the emergence of seizures after TBI.
\end{abstract}

Traumatic brain injury (TBI) is associated with secondary injuries including seizures and posttraumatic epilepsy (PTE). ${ }^{1}$ Although previous work has identified some potential biomarkers, no comprehensive study has validated biomarkers in TBI highlighting phenotypes at risk of developing seizures and/or PTE. ${ }^{2}$ Here, we report an initial assessment of the predictiveness of structural magnetic resonance imaging (MRI) biomarkers of early seizures following TBI.

\section{METHODS}

Sample

Ninety-six patients were prospectively enrolled at the time of analysis. Patients were screened/enrolled within 72 hours following a moderate-severe TBI involving frontal and/or temporal haemorrhagic contusion. Inclusion criteria were ages 6-100 and a Glasgow Coma Scale (GCS) $)^{3}$ 3-13. Exclusion criteria were isolated diffuse axonal injury, isolated epidural or subdural haemorrhages, isolated anoxic brain injury, pregnancy, incarceration and preexisting neurodegenerative or epileptic disorders. ${ }^{2}$ Although unlikely to impact group statistics, a small fraction of our cohort $(1.4 \%)^{4}$ might have had preexisting silent brain pathologies.

Of the total enrolled sample, 80 were male, 20 female and the average age was 42 years $(\mathrm{SD}=21$ years). The average field GCS was 8.29 $(\mathrm{SD}=4.45)$, closely matching the intake assessment $(\mathrm{M}=8.26, \mathrm{SD}=4.24)$. (See Vespa et $a l^{2}$ for full protocol.)

\section{Data acquisition}

For all patients we acquired, an index of injury severity (ie, GCS), continuous scalp electroencephalography (cEEG) and MRI data. As described in detail previously, 24 hours cEEG was acquired for a minimum of 72 hours during the first 7 days after TBI with a bedside 16-21 channel bipolar and referential composite montage (implemented according to each centre's intensive care unit (ICU) protocols). Minimal parameters included low frequency filter $(0.1 \mathrm{~Hz})$, high frequency filter $(50 \mathrm{~Hz})$, notch filter and $200 \mathrm{~Hz}$ sampling rate. High-resolution MRI data was acquired on $3 \mathrm{~T}$ MR systems on day 14 ( \pm 4 days) postinjury. While a panel of MR data was acquired, ${ }^{2}$ the present work focuses on the $\mathrm{T}_{1}$-weighted (MPRAGE) data which are easily acquired and translatable to nonspecialised centres. MRI parameters were optimised at each site, with a repetition time (TR) between 1.9 and $2.3 \mathrm{~ms}$, and resolution of approximately $1 \mathrm{~mm}$ isotropic or better. Higher resolution data were resampled into $1 \mathrm{~mm}^{3}$ resolution. (Previous multicentre studies have shown that variability in these parameters does not significantly impact the type of analyses performed here. $)^{56}$ Acute seizure events were recorded locally at each site, uploaded to the central Research Electronic Data Capture (REDcap) platform and reviewed by a central expert team. Longitudinal assessment for PTE was obtained at 
discharge and on days 30 and 90 postinjury with the Ottman PTE Questionnaire. ${ }^{7}$ For analysis, patients were divided into three groups: patients who experienced no seizures (no seizure group), patients who experienced at least one seizure starting by first week postinjury (early group) and patients who experienced at least one seizure starting after the first week postinjury (late group).

\section{MRI data analyses}

MR data were analysed for thinning of the cortical ribbon and subcortical local volume deficit (ie, shape analysis) following previously published procedures. ${ }^{68}$ In brief, $\mathrm{T}_{1}$-weighted data were brain extracted using optiBET ${ }^{9}$ and segmented using algorithms based on the FMRIB Software Library (FSL; for subcortical structures) ${ }^{10}$ and Freesurfer (for cortical structures). ${ }^{11}$ To ensure that results reflect local differences in ribbon thinning and/or subcortical volumes, as opposed to global head size and pose, we calculated normalised brain volume (with SIENAX) ${ }^{12}$ for each patient and used it as a normalising factor (ie, covariate) in all analyses. Cortical and subcortical segmentations were then entered in two analyses. First, we performed an analysis of variance with cortical ribbon thinning and subcortical local volume deficits (at each voxel) as dependent variables and group (no seizure, early seizure, late seizure) as the independent variable, controlling for age, sex, injury severity (ie, admission GCS), day postinjury of the MR session and normalized brain volume. Significance was assessed at $\mathrm{p}<0.05$ with familywise cluster correction for multiple comparisons. Cluster(s) showing a significant main effect of group were followed up with posthoc pairwise comparisons (with Tukey-Kramer correction). Second, we combined demographic, clinical and MR data in an analytical model to assess their relative importance in predicting vulnerability to seizures (collapsing across early and late). To reduce the dimensionality of the MR data (ie, voxels), we first extracted average shape statistics per each of 68 cortical $(34$ per hemisphere) and 15 subcortical (seven per hemisphere, plus brainstem) ROIs and entered them into a principal component analysis (with varimax rotation), retaining all components (henceforth MR components) with eigenvalue $>1$. Demographic information (ie, age, sex, days postinjury of the MR session), clinical data (admission GCS total), normalized brain volume and MR components were then entered in a binary logistic regression to distinguish patients who experienced seizure events from patient who did not. To compare the relative importance of each set of variables in predicting seizures, we perform four logistic models entering one block of variables at a time (model 1: demographic data only; model 2: demographic and clinical data; model 3: demographic, clinical and normalized brain volume; model 4: demographic, clinical, normalized brain volume and MR components).

\section{RESULTS}

Details of the final analysed sample are shown in table 1 (see Consolidated Standards of Reporting Trials flowchart, online supplementary figure S1). Of 57 patients, 22 experienced at least one seizure (early or late). While the groups did differ by sex distribution, reflecting a greater proportion of male patients, the two groups did not differ significantly in their average age, field GCS or time postinjury of the MR session. Patients who experienced at least one seizure did, however, show a marginally significant lower admission GCS total score (Welch $\mathrm{t}(51.54)=1.89$, $\mathrm{p}=0.065$ ).

As pictured in figure 1A-D, shape analysis shows significant group effect in one subcortical cluster spanning right hippocampus (cluster extent: $784 \mathrm{~mm}^{2}$, covering $33 \%$ of the right hippocampus; cluster $\mathrm{F}=4.82, \mathrm{p}=0.002$; see figure $1 \mathrm{~A}$ ) and greater cortical thinness in a right inferior temporal/fusiform cluster (cluster extent: $209 \mathrm{~mm}^{2}$, covering $2 \%$ and $7 \%$ of the inferior temporal gyrus and fusiform gyrus, respectively; cluster $\mathrm{F}=21.98, \mathrm{p}=0.001$; see figure $1 \mathrm{C}$ ). Review of initial CT findings revealed that only $17(30 \%)$ patients presented with a contusion in the right temporal lobe, suggesting that the primary injury does not sufficiently explain these results. As a comparison, more patients presented contusions in the left and right frontal lobes (21 (37\%) and $23(40 \%)$, respectively), yet neither region demonstrated significant shape change. Posthoc comparisons revealed hippocampal local volume deficit to be more pronounced in patients experiencing early seizures than patients in both other groups, whereas, at the cortical level, patients experiencing late seizures appear to have significantly larger cortical ribbon than both other groups. When cortical and subcortical ROI shape data were entered into a principal components analysis (PCA), it returned 18 components cumulatively explaining $89.8 \%$ of the total variance (see online supplementary figure $\mathrm{S} 2$ ). As shown in figure 1E (and online supplementary table S1), inclusion of brain shape data (ie, the 18 components) was key to accurately predicting susceptibility to seizures. Indeed, only the model including all variables (ie, model 4: demographic, clinical, whole brain volume and MR components) could predict with high area under the curve (AUC), accuracy, sensitivity, specificity and precision $(89 \%, 87 \%, 73 \%, 89 \%, 80 \%$, respectively) whether patients experienced seizures. Within model 4, admission GCS total $(b=-0.28, O R=0.76, p=0.02)$, the left temporal pole (PC11, see online supplementary figure $\mathrm{S} 2 ; \mathrm{b}=-1.09$,

\begin{tabular}{|c|c|c|c|c|c|}
\hline & Tot (57) & No (35) & Yes (22) & Early (14) & Late (8) \\
\hline $\operatorname{Sex}(F, M)$ & $10-47$ & $5-30$ & $5-17$ & $2-12$ & $3-5$ \\
\hline Age (years), mean (SD) & $40.46(20.31)$ & $40.45(21.62)$ & $40.49(18.53)$ & $43.41(18.56)$ & $35.38(18.52)$ \\
\hline Admission GCS total, mean (SD) & $7.91(4.184)$ & $8.69(4.41)$ & $6.68(3.55)$ & $6.93(3.29)$ & $6.25(4.17)$ \\
\hline GCS eye, med & 1 & 2 & 1 & 1 & 1 \\
\hline GCS motor, med & 4 & 5 & 3.5 & 4 & 2.5 \\
\hline GCS verbal, med & 1 & 2 & 1 & 1 & 1 \\
\hline Time post-injury (days), mean (SD) & $10.26(7.43)$ & $10.17(8.02)$ & $10.41(6.56)$ & $9.71(7.67)$ & $11.63(4.14)$ \\
\hline
\end{tabular}



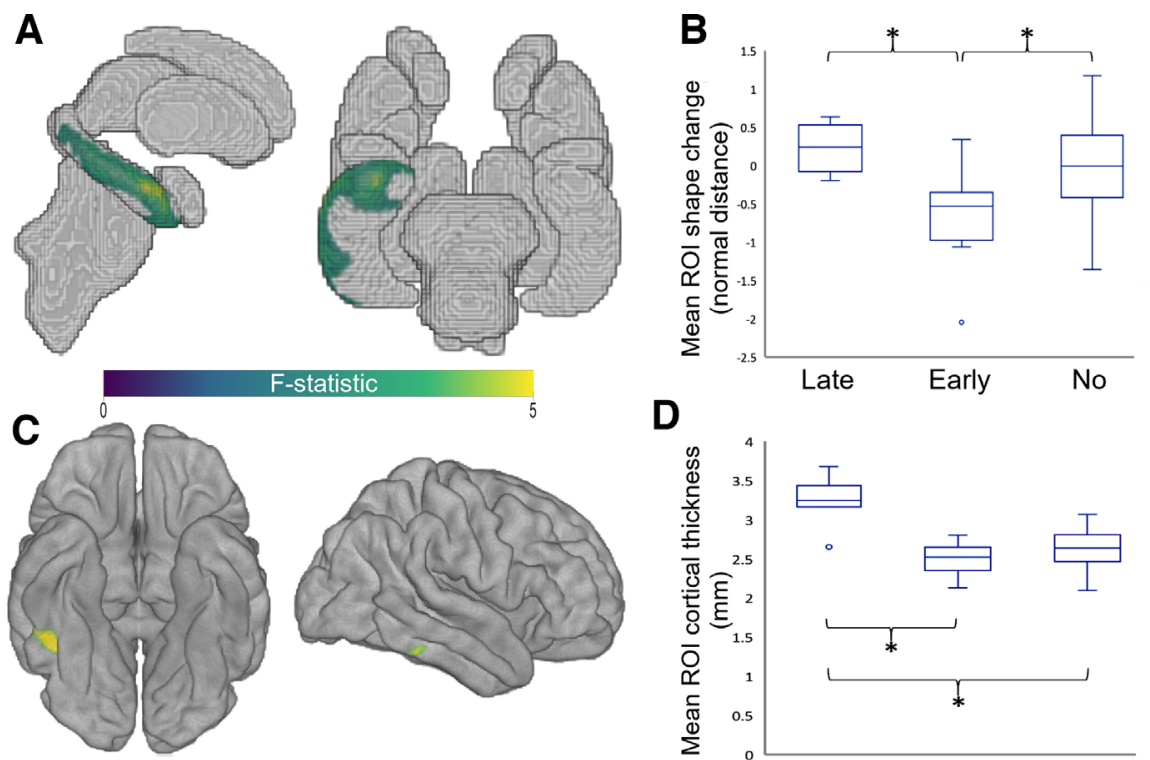

\section{D}

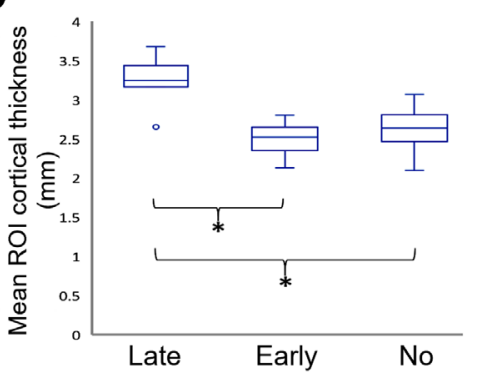

E - M1: Dem
- M2: Dem+GCS
- M3: Dem+GCS
- M4: Dem+GCS $+\mathrm{CS}_{\mathrm{T}}+$ Brain Norm Norm+Brain PCs

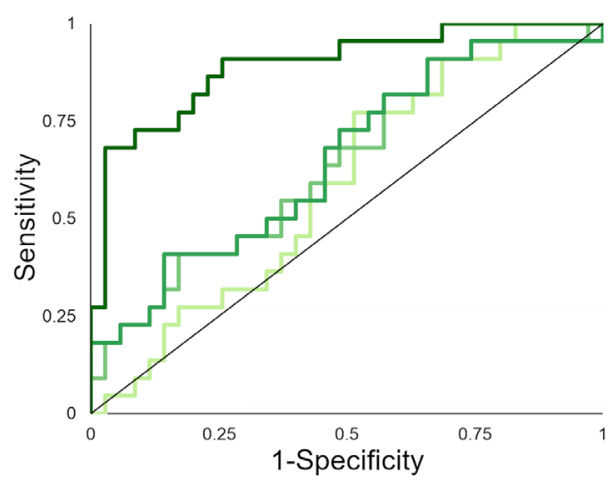

$\mathbf{F}$
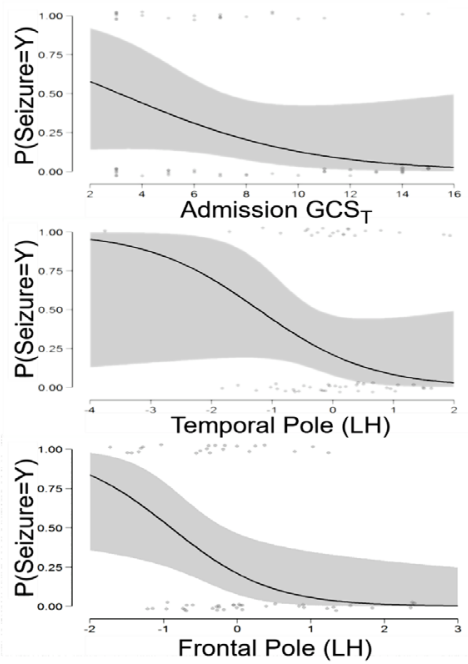

Figure 1 Results for the full brain and model-based analyses. (A) Main effect of group (F-test) for subcortical shape analysis highlighting right hippocampal local volume deficit. (B) Posthoc analysis for the hippocampal ROI found in (A) (numbers $<0$ indicate greater local volume deficit than cohort average; numbers $>0$ indicate less local volume deficit than cohort average; significance accounts for multiple comparisons with a Tukey correction). (C) Shape analysis results (F-test) for cortical regions highlighting the role of right inferior temporal and fusiform cortical thinness in differentiating across patient groups. (D) Average temporal (right inferior temporal and fusiform) cortical thickness values for the late, early and no seizures groups (significance accounts for multiple comparisons with a Tukey-Kramer correction). (E) Area under the curve comparison for the four analytical models. (F) Marginal estimated means for the three significant variables in model 4 (see text and online supplementary table S1). GCS, Glasgow Coma Scale.

$\mathrm{OR}=0.34, \mathrm{p}=0.03)$, and left frontal pole $(\mathrm{PC} 12$, see online supplementary figure $\mathrm{S} 2 ; \mathrm{b}=-1.48, \mathrm{OR}=0.23, \mathrm{p}=0.014$ ) were the three variables significantly associated with the probability of a patient experience a seizure after TBI (see figure $1 \mathrm{~F}$ ), and online supplementary table S2).

\section{DISCUSSION}

In this report, we present initial evidence of the relationship between structural abnormalities in the early acute post-TBI phase and the onset of seizures. Specifically, seizures secondary to TBI appear to be related to structural shape change in right hippocampus and temporal cortex, consistent with prior animal and human reports. ${ }^{13}$ Nonetheless, early versus late onset is associated with different patterns of structural pathology in these regions. Furthermore, we show that MR data, together with demographic data and clinical measurement of injury severity can separate with high AUC, accuracy, specificity, sensitivity and precision, patients who experience seizures from patients who do not (by 90 days postinjury). In our initial data set, injury severity and left frontal and temporal cortical thinness are the three key variables predicting vulnerability to early seizures after TBI.

As of this initial report of the EpiBioS4Rx study, ${ }^{2}$ we can only relate data to seizures occurring acutely in the ICU or in within the first 90 days. Furthermore, because the current data are based on the Ottoman questionnaire, until full review of the EEG recordings by an epileptologist, we cannot disambiguate between epileptogenicity and epileptogenesis. As the full data set is collected, we will be able to address comparative and integrative use of multiple monitoring modalities and parameters (eg, $\mathrm{T}_{2}$-weighted $\mathrm{MR}$, diffusion-weighted imaging, EEG), differentiation of acute versus longer-term 
development of seizures and evaluation of individual difference in vulnerability.

\section{Author affiliations}

'Psychology, University of California Los Angeles, Los Angeles, California, USA ${ }^{2}$ Brain Injury Research Center (BIRC), Neurosurgery, University of California Los Angeles David Geffen School of Medicine, Los Angeles, California, USA ${ }^{3}$ Laboratory of Neuro Imaging, USC Stevens Neuroimaging and Informatics Institute, University of Southern California Keck School of Medicine, Los Angeles, California, USA

${ }^{4}$ Neurology, University of California Los Angeles David Geffen School of Medicine, Los Angeles, CA, United States

${ }^{5}$ Department of Psychiatry and Biobehavioral Sciences, University of California Los Angeles, Los Angeles, CA, United States

Collaborators Agoston, Denes, Anatomy, Physiology and Genetics, Uniformed Services University of the Health Sciences; Au, Alicia K., Critical Care Medicine, University of Pittsburgh Medical Center; Bell, Michael, Critical Care Medicine, Children's National Hospital DC; Churn, Ben, NINDS, National Institute of Health; Claassen, Jan, Neurology, Columbia University; Diaz-Arrastia, Ramon, Neurology, University of Pennsylvania; Engel, Jerome Jr, Neurology, University of California Los Angeles; Foreman, Brandon, Neurology and Rehabilitation Medicine, University of Cincinnati Medical Center; Galanopoulou, Aristea, Neurology, Albert Einstein College of Medicine; Gilmore, Emily, Neurocritical Care, Yale University; Hunn, Martin, Neurosurgery, The Alfred/Monash University; Jette, Nathalie, Neurology, Icahn School of Medicine at Mount Sinai; Morokoff, Andrew, Surgery, Royal Melbourne Hospital/The University of Melbourne; Moshé, Solomon L., Neurology, Albert Einstein College of Medicine; O'Brien, Terence, Neurology, The Alfred/ Monash University/The University of Melbourne; Laing, Joshua, Neurology, The Alfred/Monash University; Perucca, Piero, Neurology, The Royal Melbourne Hospital/ Monash University; O'Phelan, Kristine H., Neurology, University of Miami; Pitkanen, Asla, A.I. Virtanen Institute for Molecular Sciences, University of Eastern Finland; Rosenthal, Eric, Neurology, Massachusetts General Hospital and Harvard University; Willyerd, Frederick, Phoenix Children's Hospital; Zimmermann, Lara, Neurology, University of California Davis Medical Center; Ellingson, Ben, Radiology, University of California Los Angeles; Buitrago Blanco, Manuel, Neurology, University of California Los Angeles; Correa, Daniel, Neurology, Montefiore Medical Center; Harrar, Dana, Pediatrics, Children's National Hospital DC; Bleck, Thomas P., Neurology, Northwestern University; Appavu, Brian, Neurology, Phoenix Children's Hospital; Struck, Aaron, Neurology, University of Wisconsin; Allen, Baxter, Neurology, Weil Cornell; Keselman, Inna, Neurology, University of California Los Angeles Health; Kennedy, Jeff, Neurology, University of California Davis Medical Center; Ferastraoaru, Victor, Neurology, Albert Einstein College of Medicine; Yoo, Ji Yeoun, Neurology, Icahn School of Medicine at Mount Sinai.

Contributors PMV, MMM and AWT conceived the study; ESL and MMM performed the analysis; CR, VS and JRT co-ordinated and performed data collection; ESL and MMM drafted the manuscript; all authors contributed critical revisions.
Funding This work was funded by NINDS Center without Walls, U54 NS100064 (EpiBioS4Rx), and by Tiny Blue Dot foundation.

Competing interests DM reports personal fees from Wiley Publishing, outside the submitted work.

Patient consent for publication Not required.

Ethics approval This work was approved by the UCLA Institutional Review Board (IRB\# 16-001 576) and the local review boards at each EpiBioS4Rx Study Group institution. Assent and written consent was obtained from the legal representative as per state law.

Provenance and peer review Not commissioned; externally peer reviewed.

ORCID iD

Martin M. Monti http://orcid.org/0000-0001-5511-3780

\section{REFERENCES}

1 Tubi MA, Lutkenhoff E, Blanco MB, et al. Early seizures and temporal lobe trauma predict post-traumatic epilepsy: a longitudinal study. Neurobiol Dis 2019;123:115-21.

2 Vespa PM, Shrestha V, Abend N, et al. The epilepsy bioinformatics study for antiepileptogenic therapy (EpiBioS4Rx) clinical biomarker: study design and protocol. Neurobiol Dis 2019:123:110-4.

3 Teasdale $G$, Jennett B. Assessment of coma and impaired consciousness. A practical scale. Lancet 1974:2:81-4.

4 Gibson LM, Paul L, Chappell FM, et al. Potentially serious incidental findings on brain and body magnetic resonance imaging of apparently asymptomatic adults: systematic review and meta-analysis. BMJ 2018;363:k4577.

5 Jack CR, Bernstein MA, Fox NC, et al. The Alzheimer's disease neuroimaging Initiative (ADNI): MRI methods. Journal of Magnetic Resonance Imaging 2008;27:685-91.

6 Lutkenhoff ES, Chiang J, Tshibanda L, et al. Thalamic and extrathalamic mechanisms of consciousness after severe brain injury. Ann Neurol 2015;78:68-76.

7 Ottman R, Barker-Cummings C, Leibson CL, et al. Validation of a brief screening instrument for the ascertainment of epilepsy. Epilepsia 2010;51:191-7.

8 Schnakers C, Lutkenhoff ES, Bio BJ, et al. Acute EEG spectra characteristics predict thalamic atrophy after severe TBI. J Neurol Neurosurg Psychiatry 2019:90:617-9.

9 Lutkenhoff ES, Rosenberg M, Chiang J, et al. Optimized brain extraction for pathological brains (optiBET). PLoS One 2014:9:e115551.

10 Patenaude B, Smith SM, Kennedy DN, et al. A Bayesian model of shape and appearance for subcortical brain segmentation. Neuroimage 2011;56:907-22.

11 Dale AM, Fischl B, Sereno MI. Cortical surface-based analysis. I. segmentation and surface reconstruction. Neuroimage 1999;9:179-94.

12 Smith SM, Zhang Y, Jenkinson M, et al. Accurate, robust, and automated longitudinal and cross-sectional brain change analysis. Neuroimage 2002;17:479-89.

13 Shultz SR, Cardamone L, Liu YR, et al. Can structural or functional changes following traumatic brain injury in the rat predict epileptic outcome? Epilepsia 2013:54:1240-50.

14 Vespa PM, McArthur DL, Xu Y, et al. Nonconvulsive seizures after traumatic brain injury are associated with hippocampal atrophy. Neurology 2010;75:792-8. 